\title{
A pilot study to evaluate assisted freehand ultrasound elasticity imaging in the sizing of early breast cancer: a comparison of B-mode and AFUSON elasticity ultrasound with histopathology measurements
}

\author{
${ }^{1}$ R E ENGLISH, MA, MBCHB, FRCR, ${ }^{2} \mathrm{~J} \mathrm{LI}, \mathrm{BSC}, \mathrm{PhD},{ }^{3} \mathrm{~A} J \mathrm{C}$ PARKER, FRCPA, MBBS(Hons), BMedSc, \\ ${ }^{3} \mathrm{D}$ ROSKELL, MA, BMBCH, FRCPath, ${ }^{1} \mathrm{R} F$ ADAMS, MA, BMBCh, FRCR, ${ }^{1} \mathrm{~V}$ PARULEKAR, MBBS, DMRD, DMRE, \\ ${ }^{1} \mathrm{~J}$ BALDWIN, MBBS, MRCP, FRCR, ${ }^{2} \mathrm{Y} C H \mathrm{I}, \mathrm{B} . \mathrm{ENG}, \mathrm{MSc}, \mathrm{PhD}$ and ${ }^{2} \mathrm{~J}$ A NOBLE, BA, DPhil, FREng
}

${ }^{1}$ Oxford Breast Imaging Centre, Oxford Radcliffe Hospitals NHS Trust, Oxford UK, ${ }^{2}$ Department of Engineering Science, University of Oxford, Oxford, UK, and ${ }^{3}$ Department of Histopathology, Oxford Radcliffe Hospitals NHS Trust, UK

Objective: This pilot study investigates the role of assisted-freehand ultrasound (AFUSON) elasticity imaging of the breast in assessing the contour, size and area of 23 early breast cancers by making comparison of AFUSON with the equivalent B-mode ultrasound images and gold standard histopathology slides.

Methods: The B-mode, AFUSON and digitised histopathology slides of three early breast cancers were compared for contour, size and area with histopathology scans. AFUSON features that corresponded to areas of known malignant change on the histopathology slides were regarded as diagnostic. These diagnostic criteria were then applied to the B-mode and AFUSON elasticity images of all 23 breast cancers in the pilot study without having the availability of the histopathology scans for reference. Corresponding diameters were measured and the results were compared with the equivalent measurements on the scans of the histology slides. The results were tabulated in histogram form. Diagnostic confidence levels were evaluated.

Results: Size dimension accuracy increased from $66 \%$ using B-mode alone to $82 \%$ using combined B-mode and AFUSON elasticity images. Tumour area accuracy was also increased. A small number of cases had a striking visual similarity of shape on AFUSON elasticity scans and histopathology slides.

Conclusion: In spite of the shortfalls in this study, AFUSON elasticity imaging was capable of acquiring some high-quality images that showed strong correlation between AFUSON elasticity and scans of histology slides. Further studies will be carried out to refine the technique and determine if it has a role in the diagnosis and management of breast cancer.

Received 24 December
2009
Revised 1 April 2010
Accepted 13 April 2010

DOI: $10.1259 / \mathrm{bjr} / 13216246$

(c) 2011 The British Institute of Radiology
Breast cancer is the most common cancer occurring in women in the Western world. Currently, the lifetime risk of developing breast cancer for UK women is 1:9, and on average 126 new cases are diagnosed each day [1]. Current trends in cancer imaging are aimed towards technological development of non-invasive techniques that provide high diagnostic sensitivity and specificity and also prognostic information about the primary tumour upon which to base treatment regimens. Such developments are taking place notably in ultrasound, CT, MRI and positron emission tomography (PET).

Ultrasound elasticity imaging of soft tissues has been developed over the last 20 years and is under evaluation as an additional tool in the ultrasound armamentarium to supplement the diagnostic information obtained during conventional B-mode scanning. It is now found

Address correspondence to: Dr Ruth English, Oxford Breast Imaging Centre, Oxford Radcliffe Hospitals NHS Trust, Old Road, Headington, Oxford OX3 7LJ, UK. E-mail: ruth.english@orh.nhs.uk Funded by the NIHR Biomedical Research Centre Programme. on several currently commercially available ultrasound systems [2-4]. These systems rely on freehand ultrasound scanning for its flexibility and simplicity [4-9] but like all freehand systems there is significant inter- and intraobserver error $[10,11]$. During the last 5 years we have developed an elasticity scanning system called assistedfreehand ultrasound (AFUSON) elasticity imaging as a method of reducing this variability by applying semiautomated, measurable external compression during an ultrasound scan. It shows improvement over freehand elasticity image acquisitions by increasing motion constraint, which translates directly into improved image quality. Improved control increases repeatability [12]. Neither freehand nor fully automated systems can accommodate these improvements while remaining sufficiently flexible for breast assessment.

Conventional freehand elasticity studies of breast cancer have largely focused on its ability to detect and classify lesions. An imaging classification and system of semi-quantification has been proposed [4] and progress has been made in the classification of masses demonstrated 
on ultrasound into those with benign and those with malignant features [4-9, 13-15], particularly Breast Imaging Reporting and Data System (BI-RADS) 3 lesions, by assessing the pattern of strain within lesions and their relative sizes on B-mode and elasticity images $[14,15]$. The little work done so far on the use of this technique as a predictor of breast cancer size when compared with the excised specimen suggests that freehand elasticity may give a more accurate pre-operative assessment than Bmode imaging [16].

Given the ability of AFUSON elasticity ultrasound to deliver increased precision and control for imaging, while maintaining the freehand operation familiar to radiologists, this pilot study was devised to test the hypothesis that AFUSON elasticity imaging could deliver a more accurate pre-operative assessment of the size, area and shape of an early breast cancer than Bmode alone. It was hoped that diagnostic criteria could be derived that would define the relationship of the cancer with the surrounding breast parenchyma.

\section{Materials and methods}

\section{Patient selection}

Patients were recruited into the study from the UK National Health Service Breast Screening Programme (NHSBSP) with full ethical approval. Patients were identified during their attendance at an NHSBSP assessment clinic. The criteria for selection were that the lesion was visible on B-mode ultrasound, that it was smaller than the footprint of the transducer and that it was likely to be a cancer on B-mode ultrasound. At the time of the diagnostic ultrasound, the patient was asked if she would be prepared to have the extra research scan. With agreement, the AFUSON elasticity study was carried out in the assessment clinic, adding about $10 \mathrm{~min}$ to the time the patient was in the department. Following the AFUSON scan all patients had a diagnostic core biopsy taken as part of routine care. Those patients whose histopathology confirmed a diagnosis of breast cancer and who were suitable for a wide local excision (WLE) were put forward into the final phase of the study. Patients requiring a mastectomy were excluded because of the technical difficulty in achieving the pathology criteria of the study protocol.

\section{Image data acquisition}

The AFUSON imaging system consists mainly of an AN2300 Digital Ultrasound Engine (Analogic Corporation, Peabody, MA), a cylinder shaped composite scanning device and a support computer (Figure 1a). The composite scanning device was designed in-house and consisted of a retractable transducer (Type 8805, B-K, Medical, Denmark) powered by a small computercontrolled linear actuator, within a cylinder, so that the transducer can perform precise axial compression when imaging (Figure 1b). The device containing the transducer was placed in contact with the skin and together the transducer and the compressor plate deformed the tissue, the extrusion of the transducer was $4.75 \mathrm{~mm}$ leading to an average tissue displacement of around $2 \mathrm{~mm}$.

Images were taken with the patient resting in the conventional supine slightly oblique position with the arm raised above the head (Figure 1a). The cylinder was supported in both hands to provide light contact with the skin and the mass was positioned in the centre of the B-mode image. Images were acquired during compression release. To prevent sag of the cylinder onto the breast a slight lifting motion was applied through the hands during the phase of probe retraction [10]. The first sagittal scan was taken at the point of maximum tumour head to toe length, a second sagittal scan was taken at the point of maximum anteroposterior thickness and an axial scan was taken through the point of maximum width in the transverse plane. An immediate post-processing tolerance graph was generated to determine that the data had been acquired within the correct pressure parameters. If the scan was outside the parameters, it was repeated until adequate images had been obtained.

B-mode and AFUSON images were acquired from the same raw data set by post-processing the ultrasound radiofrequency (RF) signal. The diagnostic AFUSON image was built from the B-mode image overlain with the coloured elasticity image using a system of transparent colours [17]. The mapping of colours was derived empirically and the colour spectrum ranged from red to blue, deep red representing very stiff tissue and deep blue representing soft tissue, leaving the background tissues grey.

The average time between the acquisition of the AFUSON images and the WLE was 40 days (range 21105 days). During this time frame, screen-detected tumours will have an undetectable growth rate [18]. Size change as a function of time was therefore ignored in this study.

\section{Pathology data acquisition}

On the day of surgery the WLE was carried out in the conventional manner. The pathology laboratory was notified that a specimen from a study patient would be arriving in the cut-up room. The pathologist arranged for the cut-up to be done in the sagittal plane, corresponding to the initial scan of the AFUSON elasticity study, thus allowing for the maximum sagittal and anteroposterior dimensions to be measured and the maximum transverse dimension to be calculated.

All pathologists were specialist breast pathologists with experience ranging from 7 to 16 years.

The slides demonstrating the relevant measurements were assessed histologically. The invasive component of the tumour contour was marked in coloured ink and any areas of in situ disease were marked in a different colour. The orientation of the specimen was noted and the margins were marked with different coloured inks. The pathologists measured the maximum anteroposterior and superoinferior dimensions by measuring the tumour on whole-mount haematoxylin and eosin (H\&E)-stained sections. The maximum mediolateral dimension was calculated by counting the number of tumour slices in 


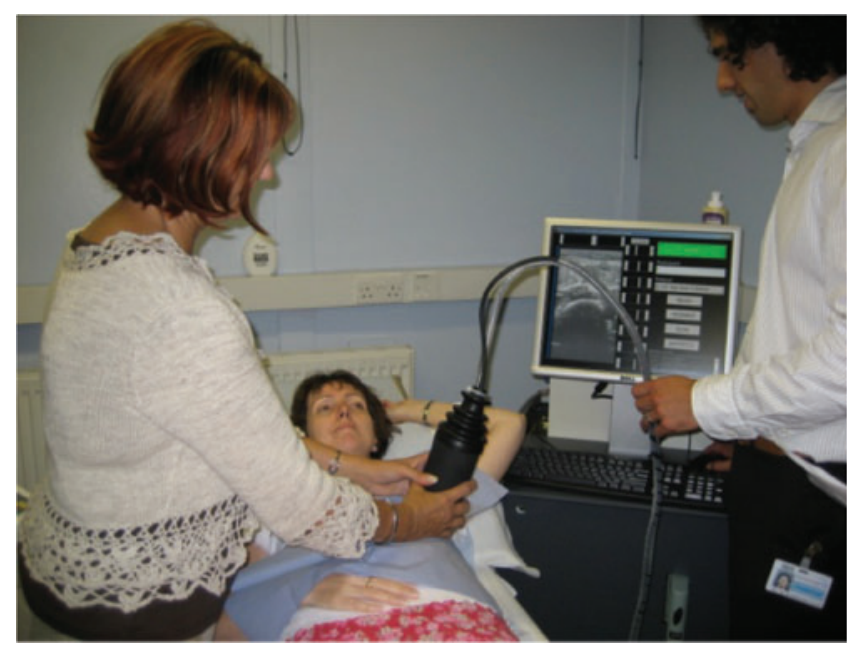

(a)

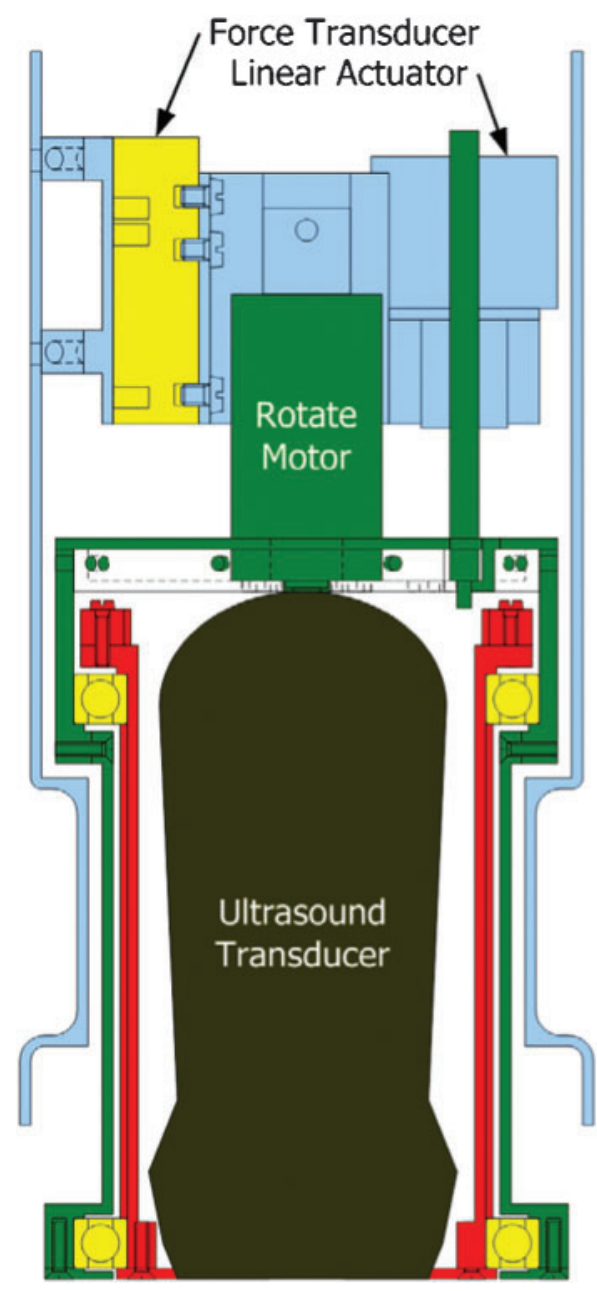

(b)

Figure 1. (a) Overview of the system. (b) Diagram of the transducer.

the cut-up and multiplying by slice thickness. The relevant slides were digitised.

\section{Data analysis}

The data were reviewed in two separate stages.

\section{Stage 1}

3 patient data sets from the total of 23 agreed for inclusion in the study were analysed. Each patient data set comprised the corresponding B-mode, AFUSON elasticity and histology slide scans displayed as parallel images on a Cintiq LCD PenTablet (Cintiq 20WSX, Wacom Technology Corporation, Vancouver, WA). Tumour contours outlined by the pathologists in colour-coded inks on the original slides prior to digital scanning enabled the orientation of the tumour on these "gold standard" images to be compared with the B-mode and AFUSON elasticity images in the same orientation. The ultrasoundacquired images were displayed at the same magnification as the pathology scans. With all information available, identical architectural features of normal breast parenchyma, tumour and peritumoural tissues were identified.
This comparison drew attention to features that might be of diagnostic value, particularly those which may differentiate benign from malignant tissue.

\section{Stage 2}

The second stage of the analysis was designed to use the diagnostic criteria derived from Stage 1 to differentiate benign from malignant tissue on the AFUSON elasticity images, without reference to the scans of the pathology slides. Because of the small number of cases eligible for inclusion in the final data set from the original number recruited into the study, the studies used in the first phase were included in the data set of the second phase. All cases were anonymised and presented in a random computer-generated order to be assessed by one radiologist (RE). Three diagnostic readings were made on each patient's data set. As the random order and the multiple sets of images generated from 23 patients for review were difficult to remember, it was felt that image recognition of the 3 patient data sets used to derive the diagnostic criteria would not cause a significant problem. It was decided not to assess interand intra-observer variation at this stage in the study. This is to be evaluated in the full study. 
- B-mode images were displayed on the LCD PenTablet. The mass was measured in three planes using standard B mode diagnostic criteria. The cancer contour was drawn on the B-mode image using a Cintiq Grip Pen on the LCD PenTablet.

- B-mode and AFUSON elasticity images were displayed side by side on the Tablet and the same measurements were repeated, taking into account the diagnostic criteria derived from Stage 1. The cancer contour was drawn on the AFUSON elasticity image at the boundary of red with yellow on the colour scale.

- The contoured B-mode and AFUSON elasticity data sets were reviewed separately by RE for confidence levels. Tumour contouring confidence was scored from 0 to 5 , with Score 0 corresponding to the least confident ( $0 \%$ confidence) and Score 5 corresponding to $100 \%$ confidence.

\section{Results}

The study recruited 83 female patients from whom 90 AFUSON elasticity studies were acquired between November 2007 and March 2009. Data sets were excluded if the histology on the core biopsy was benign, if surgery was carried out in a different hospital, if the patient required a mastectomy or if the quality of the B-mode or AFUSON images was poor. A few patients failed to be identified in the cut-up room as belonging to the study so the pathology sections did not conform to the study protocol and were therefore invalid. 23 data sets with full B-mode, AFUSON and pathology slides were suitable for inclusion in the study analysis.

\section{Patient summary}

The patient ages ranged from 51 to 70 years. The summary of data is seen in Table 1.12 patients had a palpable mass as documented in the clinical notes in the assessment clinic. 21 patients had invasive ductal carcinoma (IDC) and 2 had invasive lobular carcinoma (ILC). 20 patients had associated in situ disease. 3 patients had grade 1 disease, 11 had grade 2 and 9 had grade 3 disease. 18 were node negative, 1 had 1 positive node, 3 had 2 nodes and a patient with a grade 3 IDC had 14 positive nodes.

Data were taken from the assessment clinic notes to gather information on BI-RADS status for B-mode ultrasound and mammography. Most of the patients had BI-RADS 5 for both modalities (Table 1).

Breast density was graded as predominantly fatty, mixed fibro-fatty density or dense. 7 patients had fatty breasts, 15 had mixed density breasts and 1 had dense breasts.

Oestrogen receptor (ER) status was positive in 21 patients. Progesterone receptor (PR) was positive in 19 patients. HER2 status was not reviewed.

\section{Stage 1 results}

The first stage analysis was drawn from the three sets of images displayed side by side (Figure 2). Each image
Table 1. Patient summary (age 51-70)

\begin{tabular}{lcc}
\hline Characteristics & \multicolumn{2}{c}{ Total no. of patients (23) } \\
\cline { 2 - 3 } & IDC (21) & ILC (2) \\
\hline Palpable & 10 & 2 \\
Impalpable & 11 & 0 \\
Tumour grade & 3 & 0 \\
$\quad$ Grade 1 & 11 & 0 \\
Grade 2 & 7 & 2 \\
Grade 3 & 18 & 2 \\
Associated in situ disease & 16 & 2 \\
Node negative & 5 & 0 \\
Node positive & & \\
Ultrasound BI-RADS categories & 1 & 0 \\
3 & 2 & 0 \\
4 & 18 & 2 \\
5 & & \\
Mammography BI-RADS & & \\
categories & 2 & 0 \\
3 & 4 & 0 \\
4 & 15 & 2 \\
5 & & \\
Breast density & 6 & 1 \\
Fatty & 13 & 1 \\
Fibrofatty & 1 & 0 \\
Dense & & \\
ER/PR status & & \\
0 & $2 / 0$ & $0 / 0$ \\
4 & $0 / 2$ & $0 / 0$ \\
6 & $1 / 2$ & $0 / 1$ \\
7 & $5 / 4$ & $1 / 0$ \\
8 & $13 / 11$ & $1 / 1$ \\
\hline
\end{tabular}

IDC, invasive ductal carcinoma; ILC, invasive lobular carcinoma; ER/PR, oestrogen receptor/progesterone receptor.

was presented on the same size scale for visual comparison. Comparison of these data sets confirmed that some consistent features could be identified which were of diagnostic value.

- The strain colour map has been carefully selected according to overall tissue strain contrast between lesion and background tissue. The lesion size correlated best with the pathology sizing when using red as the lesion boundary, rather than yellow or blue.

- There is usually a linear red strain line under and following the skin surface contour (Figures 2a-f). This is because the ultrasound images were taken during compression release and the ultrasound probe was acting as the compression plate to prevent breast tissue unloading. The resulting strain image exhibits a significant stress concentration artefact, making it look stiffer near the probe-skin contact surface.

- Fibrous planes, such as ligaments of Astley-Cooper usually cross the compression direction and are less compressible due to their anisotropic mechanical property. In the corresponding strain image it may be accompanied by a red strain line marking the upper, stiffer surface (Figure 2a).

- The lesion contour most accurately resembled the pathology scan when the B-mode contour was included in the overall shape even if it did not contain any strain colouring (Figure 2e). Strain artefact may occur due to stress concentration around the lesion. 

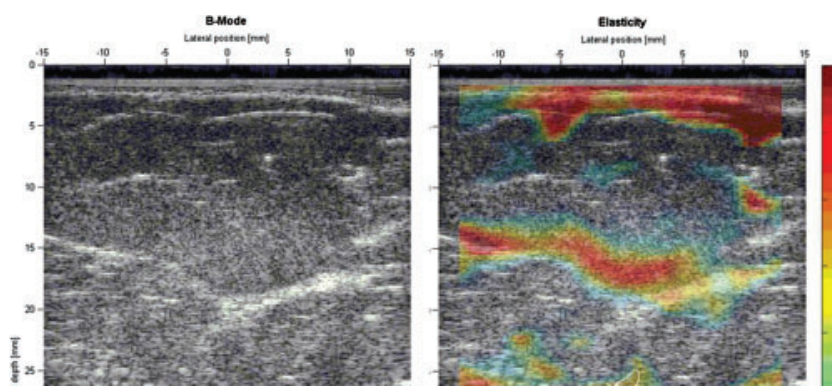

(a)
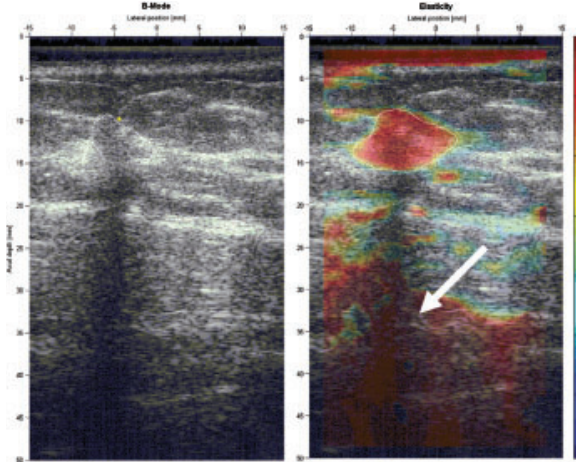

(c)
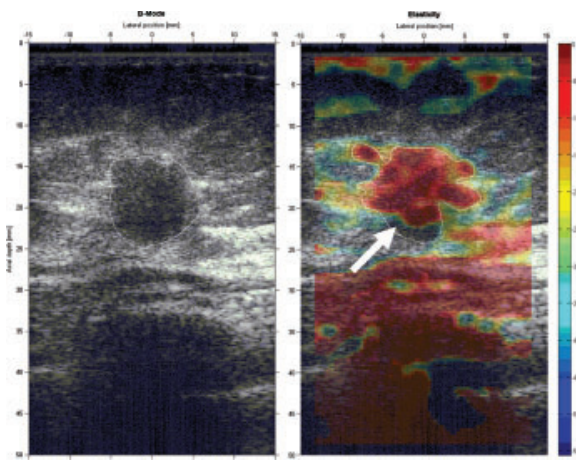

(e)
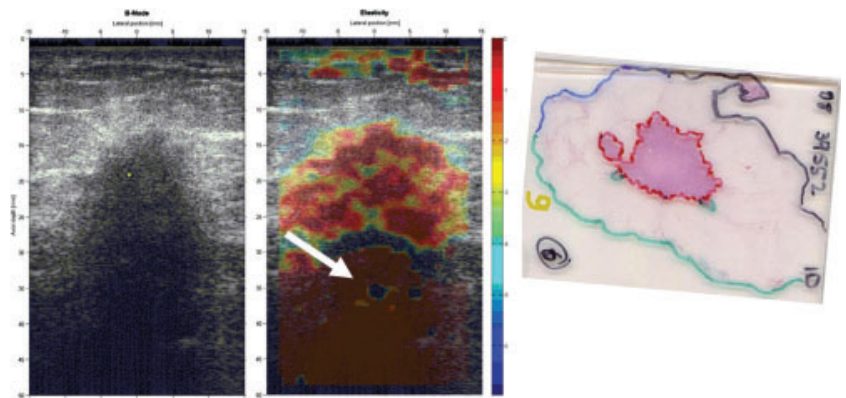
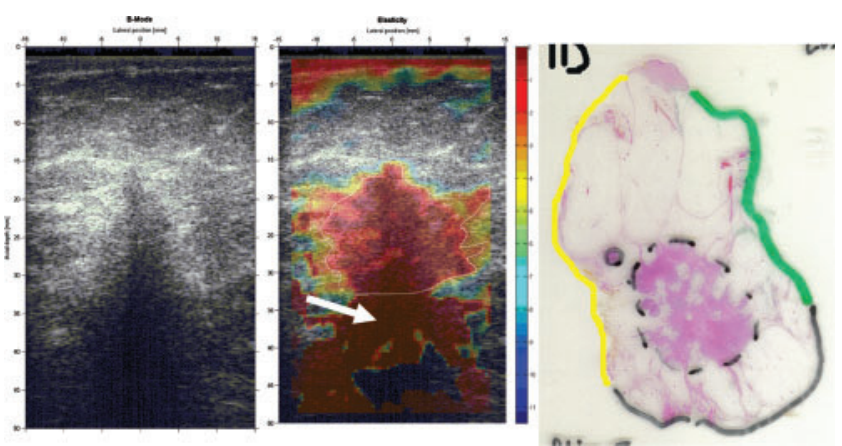

(b)
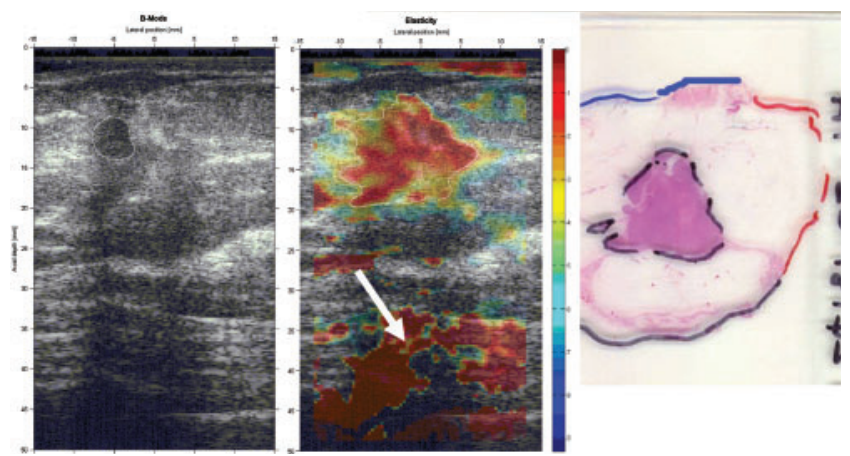

(d)
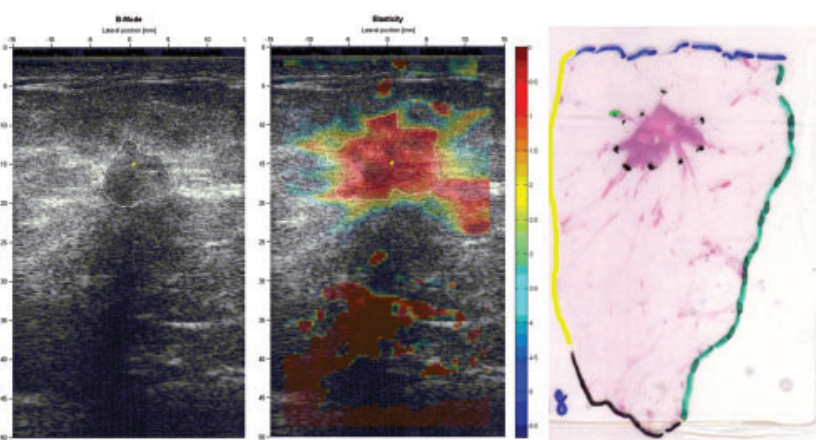

(f)

$(\mathrm{g})$

Figure 2. (a-g) Side-by-side display of ultrasound images (left), AFUSON images (middle) and wide local excision pathological slides (right). (a) Strain artefact above the fibrous planes (white arrow). (b) Shadowing artefact. (c) Strain artefact in deep region due to small tissue deformation. (d) Strain artefact in deep region due to small tissue deformation. (e) Clear lesion contour in Bmode image should be included even if no strain colouring. (f) Excluded strain artefact due to a neighbouring anatomical feature.

(g) Shadowing artefact.

Regions of red that are perceived to be due to a neighbouring anatomical feature should be excluded even if they are connected to the primary lesion (Figure 2f).
- The ultrasound signal is highly decayed by a stiff mass, showing posterior attenuation or posterior shadowing in the ultrasound image. The strain image which was derived from the ultrasound radiofrequency 
signal before and after compression cannot give meaningful tissue stiffness information at these very low signal-to-noise ratio areas. A stiff mass may have an area of depleted or disorganised strain pattern posteriorly obscuring the posterior boundary (Figure $2 \mathrm{~b}$ ).

- Owing to stress decay with respect to depth, in deep regions the tissue deformation is very small. The strain image reveals considerable artefact that obscures the real stiffness distribution. The deeper the lesion, the less likely it is to show a discrete strain pattern (Figure $2 \mathrm{c}$ and $\mathrm{d}$ ).

\section{Stage 2 results}

The second stage analysis was designed to investigate if the above diagnostic criteria confirmed the hypothesis that the dimensions obtained from the AFUSON elasticity images were more similar to the gold standard pathology dimensions than B-mode alone. Three tumour measurements were made on each scan, i.e. sagittal (longitudinal), sagittal (anteroposterior) and axial (width left to right) for the three data sets. In this study most of the tumour sizes range between 1 and $2 \mathrm{~cm}$ and their histological dimensions are shown in Table 2. The histology measurements of tumour width were indirect, taken from the number of macroscopic pathology slices multiplied by their thickness. This was felt to be less accurate than the other two planes and therefore was not included in the analysis. The distribution of tumour size using B-mode image and AFUSON elasticity image in comparison with histology is shown in Figure 3a (longitudinal dimension) and Figure $3 b$ (anteroposterior dimension). The gold standard dimension was plotted as zero on the graph. The bars above and below zero represent the deviation of the measurements from the gold standard pathology scans. As can be seen, the tumour sizes measured on the AFUSON images is closer to the gold standard than B-mode alone. Analysis of the data shows that the measurement accuracy is increased from $66 \%$ using B-mode alone to $82 \%$ by combining B-mode image and AFUSON images together.

As well as tumour dimensions, tumour area was calculated from the manually contoured ultrasound B-mode images, the AFUSON elasticity image and the histology scans. Figures $4(\mathrm{a}, \mathrm{b})$ shows the tumour area deviation using ultrasound B-mode image and AFUSON image from the gold standard histology measurements. Tumour area measurement error was most reduced on the AFUSON elasticity image.

Tumour size measurement confidence was shown in Table 3. Confidence levels were divided into 6 scores, Score 0 corresponding to minimal confidence and Score 5

Table 2. Distribution of tumour size according to histology

\begin{tabular}{lcc}
\hline \multirow{2}{*}{ Tumour size } & \multicolumn{2}{l}{ Number of cases } \\
\cline { 2 - 3 } & Longitudinal dimension & Anteroposterior dimension \\
\hline$<1 \mathrm{~cm}$ & 4 & 5 \\
$1-1.5 \mathrm{~cm}$ & 11 & 10 \\
$1.5-2 \mathrm{~cm}$ & 6 & 7 \\
$>2 \mathrm{~cm}$ & 2 & 1 \\
\hline
\end{tabular}

corresponding to the highest level of confidence. Confidence scores were recorded for both the B-mode image alone and the AFUSON image. The mean confidence scores were calculated by averaging the total confidence score over the number of patients for each type of tumour. The overall confidence measurement increases slightly when using the AFUSON images. The confidence measurement for a tumour without ductal carcinoma in situ (DCIS) increases significantly in comparison with tumours that have surrounding DCIS, both on B-mode and AFUSON imaging.

Figure 5 shows mean tumour size measurement error with respect to measurement confidence. Measurements from strain images show higher accuracy in all but one confidence score. In this one case the confidence score was 5 so no conclusion can be drawn. The results also show that when confidence is low (Score 2 and 3), tumour size measurements by strain imaging performed much better than by B-mode imaging, with the deviation decreased by $22 \%$.

\section{Discussion}

This study was designed to investigate the relationship between size, area and shape of a breast cancer using AFUSON elasticity imaging, a novel ultrasound elasticity technique. We examined the hypothesis that the presence of malignant cells in breast parenchyma would change the elastic properties of that tissue thereby being a useful indicator of the presence malignant invasion that is occult on mammography and B-mode ultrasound. The practical importance of this is that it may be possible to achieve a more accurate pre-operative sizing, allowing a one stage surgical procedure. Currently about 20\% [19] of patients diagnosed on the NHSBSP who undergo a treatment operation require a second procedure, some of which are to remove margins involved by tumour not removed in the primary operation. This percentage could be reduced with more accurate pre-operative estimation of tumour size and shape.

To our knowledge this study is the first of its type to attempt semi-automated elasticity imaging in the direct comparison of imaging acquired size and contour data with histology sections as the gold standard. Such a study has inherent difficulties in attempting the exact replication of a ground truth by two unrelated techniques and this study is no exception.

We had to make the assumption that the maximum dimensions taken in any one plane on ultrasound corresponded to the maximum dimensions measured on the scan of the histology slide. It may be that if the tumour was occult on ultrasound at a given point, then the maximum histological measurement could be at another location in the tumour. For the purposes of this study it was assumed that the maximum dimensions on AFUSON and histology were concordant.

The ultrasound research machine used in the study was of an older generation and the B-mode image did not have as high a resolution as the newer generation of machines. A source of concern during the study was the accuracy of the B-mode measurements. However, there was no other ultrasound machine available to us that would allow access to the RF signal for post-processing 


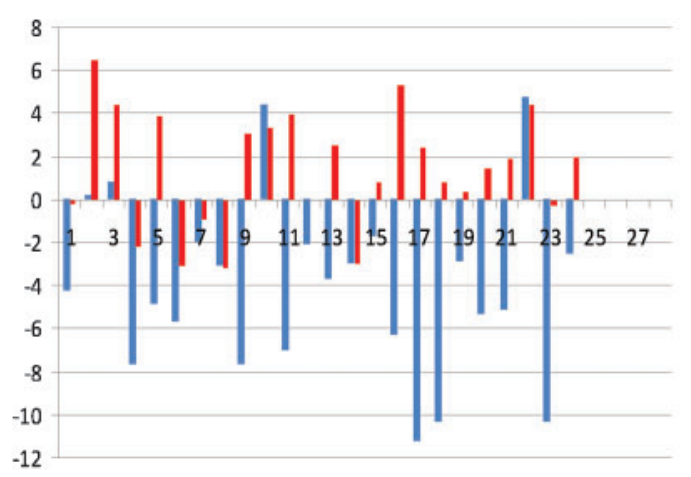

(a)

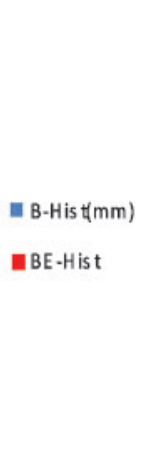

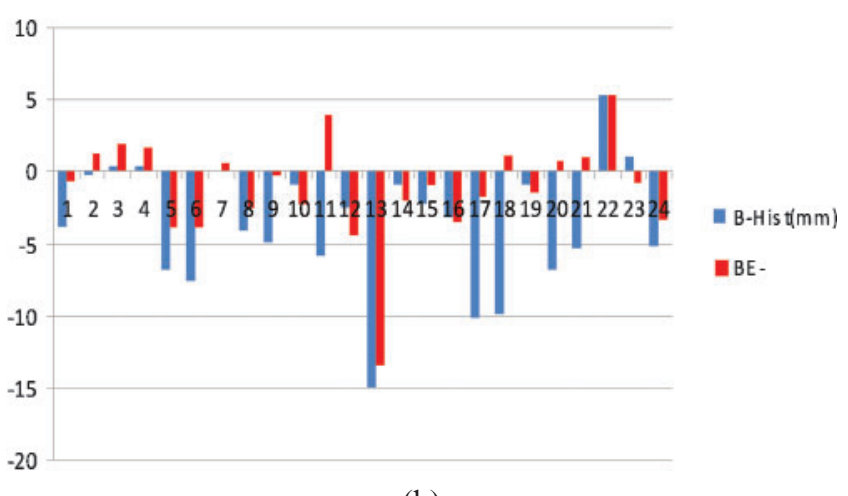

(b)

Figure 3. Comparison of tumour size measurement by using ultrasound image alone (blue bar) and AFUSON image (red bar). (a) The tumour size measurement error from sagittal longitudinal plane and (b) the error from axial anteroposterior plane measurement.

the image. A newer generation machine will be available for the full study that has a sharper B-mode display and also allows access to the RF signal.

A possible source of error in the data was that the cutup may not have been done precisely in the correctly orientated plane. In addition, it is well recognised that the processing of histological samples causes shrinkage. The literature gives wide variability in the degree of shrinkage of both the tumour and the specimen margin [19]. The measurements taken from the pathology slides that were used as our gold standard were calculated to take an overall 10\% shrinkage into account.

The characteristics of patient selection were largely typical of those recruited through the NHSBSP although there were a slightly higher number $(87 \%)$ of grade 2 and 3 cancers [20] (Table 1) than in the average screendetected population (72\%). Only two of the patients had invasive lobular carcinoma, so tumour type was not correlated with the outcomes. ER and PR status and breast density were recorded but their effect was not evaluated in this study.

The technical innovation in this study is in the design of the hand-held device and its usage to mimic a laboratory gantry. Instead of the operator performing the compression, the device is held stationary. In this manner, the device operates in a freehand manner, but with assistance provided whenever and wherever a platform is established. To improve strain image quality and reduce estimator variance, it is important to minimise stress concentrations (these occur due to elasticity variations and compression boundary conditions); therefore, a compressor contact surface larger than the imaging window while keeping the flexibility afforded by freehand imaging is desirable. Normal commercial transducers vary from $30 \mathrm{~mm}$ to $60 \mathrm{~mm}$ in width, thus a compressor plate twice the width of the small transducer face $(\sim 70 \mathrm{~mm})$ should still be manageable whilst minimising stress concentrations and was used in this study. Patients found the scanning technique very acceptable with a short acquisition time and overall comfort in positioning.

Rules for elasticity image interpretation were derived using the observations on tissue characteristics as described above. It was apparent that most information was obtained when B-mode and AFUSON elasticity images and pathology scans were viewed side by side at the same scale of magnification.

The critical diagnostic differentiation to be made was between benign stiff tissue and malignant disease. A good example of this is the appearance of ligaments of Astley-Cooper. In Figure 2a, a strong red bar is seen above the hyperechoic plane of the ligament on the superimposed B-mode image. This principle of using a bar of stiffness on elasticity imaging which corresponded to a known normal anatomical feature on the B-mode scan as an indicator of benign (Figure 2f) enabled a boundary to be drawn between benign and malignant tissue in an area of uniform redness on the AFUSON elasticity image.

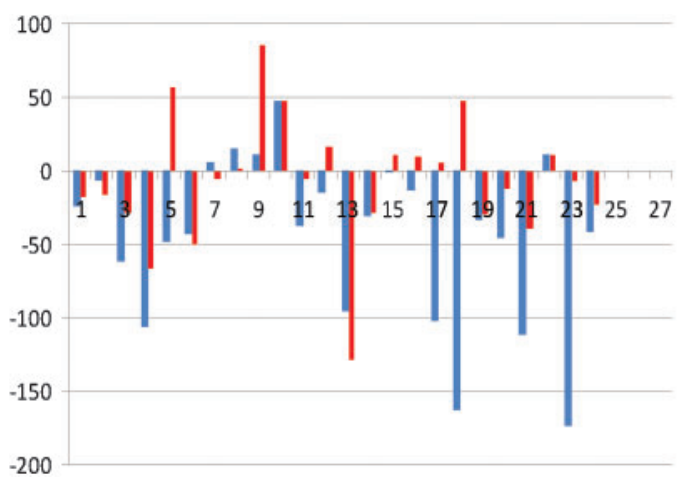

(a)

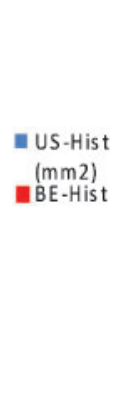

$\left(\begin{array}{l}(\mathrm{mm} 2) \\ \mathrm{BE}-\mathrm{Hist}\end{array}\right.$

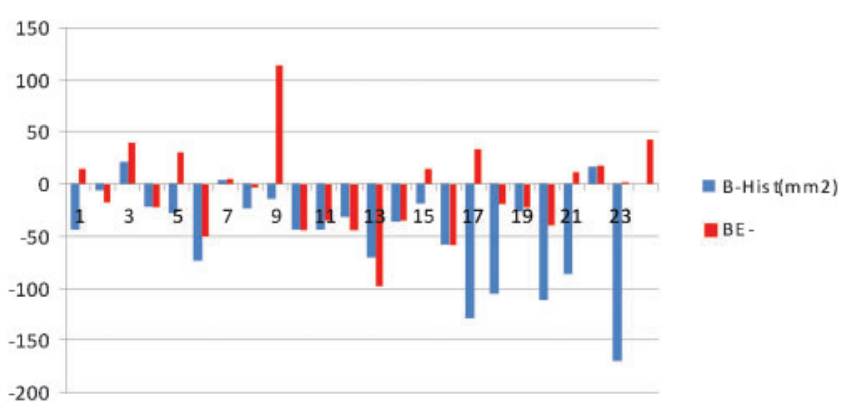

(b)

Figure 4. Comparison of tumour area measurement by using ultrasound image alone (blue bar) and by AFUSON image (red bar). (a) The tumour area measurement error from sagittal longitudinal plane and (b) the error from axial anteroposterior plane measurement. 
Table 3. Mean tumour size measurement confidence score (score $0=0 \%$ confidence and score $5=100 \%$ confidence, $n$ is the number of patients)

\begin{tabular}{lll}
\hline Classification $(\boldsymbol{n})$ & \multicolumn{2}{l}{ Mean confidence score } \\
\cline { 2 - 3 } & B & B+BE \\
\hline Malignant in total (23) & 2.91 & 3 \\
Ductal carcinoma (21) & 2.90 & 3.05 \\
With DCIS (18) & 2.78 & 2.94 \\
Without DCIS (3) & 3.67 & 3.67 \\
Lobular carcinoma (2) & 3 & 2.5 \\
Palpable (11) & 3.27 & 3.72 \\
Non-palpable (12) & 2.58 & 2.33 \\
\hline
\end{tabular}

DCIS, ductal carcinoma in situ.

Conversely, some areas of the B-mode images where conventional interpretation lead to the diagnosis of probable malignancy, failed to show red colouration on the elasticity image (Figure 2a). It was thought to be reasonable to include such areas within the diagnostic elasticity tumour boundary.

The posterior margin of the tumour was the most difficult to identify on both B-mode and AFUSON scans because of decay of the primary ultrasound signal (Figure 2b). An attempt to define the posterior boundary was made using adjacent B-mode anatomical features such as the anterior surface of pectoralis major or ligaments of Astley-Cooper. However front to back dimensions on both images remained unsatisfactory in some cases. Overall, cancer boundaries were more easily seen in superficial lesions than deeper placed lesions in which there was stress decay in regions where tissue deformation was small.

Several tumours showed a wide surrounding halo of colour graduation from deep red to pale blue (Figure 2f). The elasticity sizing correlated best with the pathology dimensions when the red boundary was selected.

Some tumours had a mixed red/yellow interior (Figure 2d). In these cases, the yellow was included in the elasticity tumour boundary.

When all the data sets were analysed in Phase 2 of this pilot study using these criteria, the results suggest that there is improved correlation between the tumour dimensions and area when using combined B-mode and

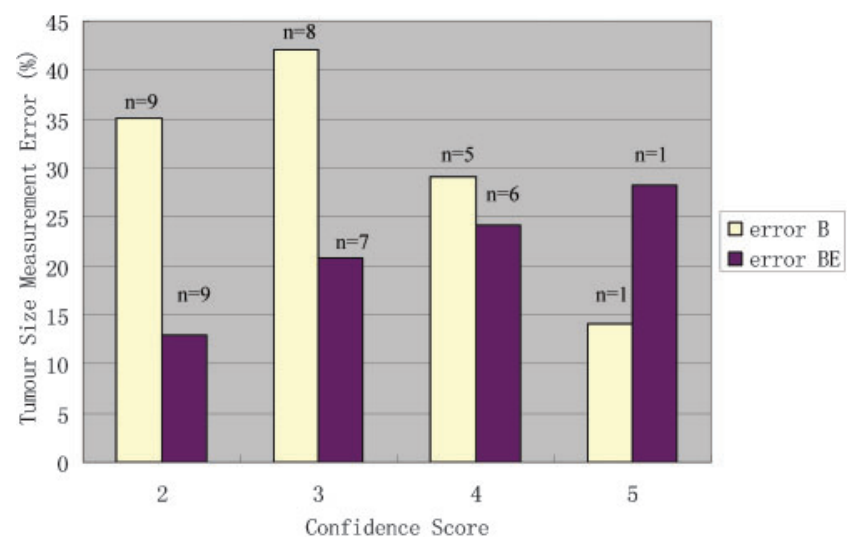

Figure 5. Distribution of tumour size measurement error with respect to confidence by using ultrasound image alone (yellow bar) and by AFUSON image (purple bar). $\mathrm{n}$ is the patient code number.
AFUSON elasticity rather than B-mode alone. The final data analysis suggested that diagnostic confidence is somewhat increased.

However, these percentage differences are small and given the small numbers in this pilot study no great significance can be attributed to the figures. Many of the original studies were discarded, mainly because the core biopsy histology was benign, the surgery was carried out at a different centre, the patient required a mastectomy or the cut-up did not conform to the study protocol. Only a few data sets were excluded, some because the elasticity images were not interpretable but we did attempt to include data sets that were of less than ideal quality. Nevertheless, and most importantly, we found there to be a striking and strong visual correlation in a small number of cases between the contour of the tumour on the scan of the pathology slide and its contour on the AFUSON elasticity image. It is on the basis of these cases, rather than the overall measurements, that we believe there to be a case for further investigating if AFUSON elasticity imaging may be a useful tool in the pre-operative assessment of breast cancer size. Further studies could include the investigation of the intra- and interobserver variation of AFUSON image assessment by several radiologists. An AFUSON elasticity imaging system that uses a new generation B-mode ultrasound scanner is now under development and the comparison of AFUSON with freehand elasticity imaging in this context would be favourable to study.

\section{Conclusion}

This pilot study produced some striking AFUSON images that showed a high visual correlation with the pathology scan. The shortfalls and problems encountered in this pilot study have been discussed. Nevertheless, the ability of AFUSON elasticity imaging to acquire highquality tumour images showing a striking similarity to the histological tumour contour in a small number of patients has been demonstrated. In addition to increased image quality, the tumour dimensions as measured on AFUSON elasticity imaging have a higher accuracy than B-mode alone for comparing histopathology measurements. Further studies will be carried out to develop and evaluate AFUSON to determine if it has a role in breast cancer management.

\section{Acknowledgments}

Dr C Hughes, Dr A Maher and Dr I Low for their help in reporting some of the scanned histopathology slides.

\section{References}

1. Cancer Research UK. CancerStats Breast Cancer-UK. London, UK: Cancer Research. 2009;1-2.

2. Scaperrrotta G, Ferranti C, Costa C, Mariani L, Marchesini M, Suman L, et al. Role of sonoelastography in non-palpable breast lesions. European Radiology 2008;18:2381-9.

3. Tan SM, Teh HS, Mancer JF, Poh WT. Improving B mode ultrasound evaluation of breast lesions with real-time ultrasound elastography-A clinical approach. Breast 2008;17: 252-7. 
4. Itoh A, Ueno E, Tohno E, Kamma H, Takahashi H, Shiina T, et al. Breast disease: clinical application of US elastography for diagnosis. Radiology 2006;239:341-50.

5. Garra BS, Cespedes EI, Ophir J, Spratt SR, Zuurbier RA, Magnant CM, Pennanen MF. 1997; Elastography of breast lesions: initial clinical results. Radiology 1997;202: 79-86.

6. Hiltawsky KM, Kruger M, Starke C, Heuser L, Ermert H, Jensen A, et al. Ultrasound Med Biol 2001;27:1461-9.

7. Bamber JC, Barbone PE, Bush NL, Cosgrove DO, Doyely MM, Fuechsel FG, et al. Progress in freehand elastography of the breast. IEICE Trans Inf Syst 2002;85:5-14.

8. Svennson WE, Amiras D, Shousha S, Rattansigh A, Chopra D, Sinnett HD, et al. Elasticity imaging of 67 cancers and 167 benign breast lesions shows that it could halve biopsy rates of benign lesions. In: Proceedings of the 4th International Conference on Ultrasonic Measurement and Imaging of Tissue Elasticity. Austin TX, 2005;16-19.

9. Regner DM, Helsey GK, Hangiandreou MJ, Morton MJ, Nordland MR, Meixner DD, et al. Breast lesions: Evaluation with US strain imaging-clinical experience of multiple observers. Radiology 2006;238:425-37.

10. Hall TJ, Zhu Y, Spalding CS. In vivo real-time freehand palpation imaging. Ultrasound Med Biol 2003;29:427-35.

11. Chandrasekhar T, Ophir J, Krouskop K, Ophir K. Ultrasound Med Biol 2006;32:847-55.

12. Kadour M, Noble JA. Assisted-freehand ultrasound elasticity imaging. IEEE Transactions on Ultrasonics, Ferroelectrics and Frequency Control 2009;56:36-43.
13. Burnside E, Hall T, Sommer AM, Helsey GK, Sisney GA, Svenssen WE, et al. Differentiating benign from malignant solid breast masses with US strain imaging. Radiology 2007; 245:401-10.

14. Chandra M, Sehgal S, Weinstein E, Arger PH, Conant EF. A review of breast ultrasound. J Mammary Gland Biol Neoplasia 2006;11:113-23.

15. Elenbaas B, Weinberg RA. Heterotypic signalling between epithelial tumor cells and fibroblasts in carcinoma formation. Exp Cell Res 2001;264:169-84.

16. Svennson W, Wakeham NR, Usupbaeva A, Shousa S, Tydeman CAJ, Cosgrove DO, et al. Ultrasound elasticity imaging is more accurate than B-mode ultrasound for assessing the extent of invasive cancers-early results of pathological correlation. Ultrasound Med Biol 2006;32:173.

17. Kadour M. Assisted-freehand ultrasound elasticity imaging. PhD Thesis 2007, University of Oxford.

18. Weedon-Fekjaer H, Lindqvist BH, Vatten LJ, Aalen OO, Tretli S. Breast cancer tumor growth estimate through mammography screening data. Breast Cancer Research 2008; http//breast-cancer-research.com/content/10/3/R41

19. Yeap BH, Muniandy S, Lee S-K, Sabaratnam S, Singh M. Specimen shrinkage and its influence on margin assessment in breast cancer. Asian J Surg 2007;30:183-7.

20. West Midlands NHS Breast and Screening Quality Assurance Reference Centre. NHS Breast Screening Program and Association of Breast Surgery at BASO. An audit of screen detected breast cancers for the year of screening April 2007-March 2008: 9-32. 